\title{
Magnetic susceptibility of quark matter within Fermi-liquid theory
}

\author{
T. Tatsumi and K. Sato \\ Department of Physics, Kyoto University, Kyoto, 606-8502, Japan
}

\begin{abstract}
Possibility of spontaneous magnetization in QCD and magnetic properties of quark matter is discussed by evaluating the magnetic susceptibility within Fermi-liquid theory. The screening effects for gluons are taken into account to figure out the specific properties of the magnetic transition in gauge theories. It is shown that the static screening effect in terms of the Debye mass does not necessarily work against the magnetic instability; it promotes the instability, depending on the coupling constant and the number of flavors.
\end{abstract}

Many efforts have been devoted to explore the QCD diagram on the density $\left(\rho_{B}\right)$ - temperature $(T)$ plane, which is closely related to relativistic heavy-ion collisions, early universe and compact stars [1. In particular, it would be interesting to consider the low temperature case, where quark degrees of freedom are dominant over gluons and the concept of the Fermi sea is well defined. Various correlations are expected to be realized there as in condensed-matter physics; actually there have been many works about color superconductivity at high-density quark matter [1].

A possibility of spontaneous spin polarization in quark matter has been suggested by one of the authors [2], using the one-gluon-exchange (OGE) interaction. The onset mechanism is very simple but it reflects an interesting aspect of magnetic phase transition in gauge theories as QED or QCD [2, 3, 4, 5]. The direct term gives a null contribution due to the charge (color) neutrality, so that the leading-order contribution comes from the exchange term. The Fock exchange interaction then favors the spin alignment due to 
the Pauli principle and its infinite-ranginess gives a particular density dependence of the exchange term. If it is realized, it may give a microscopic origin of strong magnetic field observed in compact stars, especially magnetars [6].

In this work we further explore the properties of the magnetic phase transition in QCD by examining the magnetic properties of quark matter in a paramagnetic phase, by the use of Landau Fermi-liquid (FL) theory [7, 8]. We discuss magnetic susceptibility in detail to figure out the critical behavior of the magnetic phase transition in gauge theories at finite density. We only consider here the normal phase of quark matter as a first step for fully understanding the coexistence of magnetic and superconducting phases.

It is well-known that there appear infrared (IR) singularities in the FL interactions in the gauge theories. To improve the IR behavior of the gauge interactions it is necessary to take into account the screening effects for the gauge field [4, 7]. The inclusion of the screening effects is also required by the argument of the HDL resummation [9]. Since the IR singularities appear for quasi-particles with the co-linear momenta, the soft gluon should give a dominant contribution. Then the particle-hole polarization function $\sim$ $O\left(g^{2} \mu^{2}\right)$ should be the same order of magnitude with the energy-momentum of soft gluons $\sim O(g \mu)$, where $g$ and $\mu$ are the QCD coupling constant and quark chemical potential, respectively. For the longitudinal gluons we can see the static screening by the Debye mass and the IR behavior is surely improved. However, there is no static screening for the transverse gluons and there is only the dynamic screening. Thus the IR singularities are still left for the interactions through the exchange of the transverse gluons.

Applying the uniform but tiny magnetic field on quark matter, one may evaluate magnetic susceptibility defined as

$$
\chi_{M} \equiv \sum_{f=u, d, s} \chi_{M}^{f}=\left.\sum_{f=u, d, s} \frac{\partial\langle M\rangle_{f}}{\partial B}\right|_{B=0}
$$

with the magnetization $\langle M\rangle_{f}=V^{-1}\left\langle\mu_{q} \int d^{3} x \bar{q}_{f}\left[(-i \mathbf{r} \times \nabla)_{z}+\Sigma_{z}\right] q_{f}\right\rangle$, where we take $\mathbf{B} / / \hat{\mathbf{z}}$. In the following we consider the isotropic quark matter, so that the first orbital contribution can be discarded and only spin degrees of freedom contributes to magnetization. Since gluons only carries color, we can consider the partial $\chi_{M}^{f}$ for each flavor 1 . The inverse of $\chi_{M}^{f}$ measures curvature of the free energy $F\left(\langle M\rangle_{f}\right)$ at the origin, $\left(\chi_{M}^{f}\right)^{-1}=d^{2} F /\left.d\langle M\rangle_{f}^{2}\right|_{\langle M\rangle_{f}=0}$,

\footnotetext{
${ }^{1} \mathrm{We}$, hereafter, omit the subscript to specify the flavor for simplicity.
} 
so that the divergence of $\chi_{M}^{f}$ signals the spontaneous spin polarization. The spin degrees of freedom of a quark with momentum $\mathbf{k}$ is specified by the spin vector $a_{\mu}(\mathbf{k})\left[2, \mathbf{a}(\mathbf{k})=\zeta\left[\hat{\mathbf{z}}+\mathbf{k}(\hat{\mathbf{z}} \cdot \mathbf{k}) / m\left(E_{k}+m\right)\right], a^{0}(\mathbf{k})=\zeta \hat{\mathbf{z}} \cdot \mathbf{k} / m\right.$ with $E_{k}=\left(\mathbf{k}^{2}+m^{2}\right)^{1 / 2}$ and $\zeta= \pm 1$. We consider the static susceptibility for a constant magnetic field along $z$ - axis. Each Fermi sea for $\zeta= \pm 1$ is a little changed in the presence of the magnetic field. Then magnetization may be given by the difference in the number densities $\delta n_{\mathbf{k}}^{a}=n_{\mathbf{k}, \zeta=1}-n_{\mathbf{k}, \zeta=-1}$ with the distribution function, $n_{\mathbf{k}, \zeta}=\left[\exp \beta\left(\epsilon_{\mathbf{k}, \zeta}-\mu-1 / 2 g_{D}(\mathbf{k}) \mu_{q} \zeta B\right)+1\right]^{-1}$, where $\epsilon_{\mathbf{k}, \zeta}$ is the quasi-particle energy in the presence of the external magnetic field 2. Thus we find

$$
\langle M\rangle=\frac{\mu_{q}}{2} N_{c} \int \frac{d^{3} k}{(2 \pi)^{3}} g_{D}(\mathbf{k}) \delta n_{\mathbf{k}}^{a}
$$

where $\mu_{q}$ denotes the Dirac magnetic moment, $\mu_{q}=e_{q} / 2 m$, and the gyromagnetic ratio $g_{D}(\mathbf{k})$ is defined as $g_{D}(\mathbf{k}) \equiv 2\left(a_{z}-k_{z} / E_{k} a_{0}\right)=2\left[1-k_{z}^{2} / E_{k}\left(E_{k}+\right.\right.$ $m)]$. For the weak magnetic field, dynamics among the quasi-particles around the Fermi surface determine the response to the external magnetic field. Actually we find that the number-difference arises as a result of change of the quasi-particle energy around the Fermi surface,

$$
\begin{aligned}
\delta n_{\mathbf{k}}^{a} & =\sum_{\zeta} \frac{\partial n_{\mathbf{k}}}{\partial \epsilon_{\mathbf{k}}}\left(\epsilon_{\mathbf{k}, \zeta}-\epsilon_{\mathbf{k}}\right) \\
& =\frac{\partial n_{\mathbf{k}}}{\partial \epsilon_{\mathbf{k}}}\left[-g_{D}(\mathbf{k}) \mu_{q} B+N_{c} \sum_{\zeta, \zeta^{\prime}} \zeta \int \frac{d^{3} q}{(2 \pi)^{3}} f_{\mathbf{k} \zeta, \mathbf{q} \zeta^{\prime}} \delta n_{\mathbf{q}, \zeta^{\prime}}\right],
\end{aligned}
$$

where $\epsilon_{\mathbf{k}}$ denotes the quasi-particle energy in the absence of the magnetic field. Here the first term imply the explicit $B$ dependence of the quasiparticle energy, while the second term is a rearrangement term peculiar to FL theory and represent the implicit change of the quasi-particle energy through the change of the distribution function, $\delta n_{\mathbf{q}, \zeta} \equiv n_{\mathbf{q}, \zeta}-n_{\mathbf{k}}$. Thus we may see that some non-perturbative effects are taken into account in Eq. (3) in a self-consistent manner, even if we treat the quasi-particle interaction $f_{\mathbf{k} \zeta, \mathbf{q} \zeta^{\prime}}$ in a perturbative way. The quasi-particle interaction consists of two parts, the spin independent $\left(f_{\mathbf{k}, \mathbf{q}}^{s}\right)$ and independent $\left(f_{\mathbf{k}, \mathbf{q}}^{a}\right)$ terms;

$$
f_{\mathbf{k} \zeta, \mathbf{q} \zeta^{\prime}}=f_{\mathbf{k}, \mathbf{q}}^{s}+\zeta \zeta^{\prime} f_{\mathbf{k}, \mathbf{q}}^{a}
$$

\footnotetext{
${ }^{2}$ Generally topology of the Fermi surface is modified due to the spin-momentum coupling, but one may safely discard it in the weak-field limit.
} 
From Eqs. (2),(3) magnetic susceptibility is written in terms of the FL interaction,

$$
\chi_{M}=\left(\frac{\bar{g}_{D} \mu_{q}}{2}\right)^{2} \frac{N(T)}{1+N(T) \bar{f}^{a}}
$$

with $\bar{g}_{D} \equiv \int_{|\mathbf{k}|=k_{F}} d \Omega_{\mathbf{k}} / 4 \pi g_{D}(\mathbf{k}) . \quad \bar{f}^{a}$ is an angle-averaged Landau-Migdal parameter, which measures the strength of the spin dependent interaction,

$$
\left.\bar{f}^{a} \equiv \int \frac{d \Omega_{\mathbf{k}}}{4 \pi} \int \frac{d \Omega_{\mathbf{q}}}{4 \pi} f_{\mathbf{k}, \mathbf{q}}^{a}\right|_{|\mathbf{k}|=|\mathbf{q}|=k_{F}}
$$

at $T=0$.

$N(T)$ is the effective density of states at the Fermi surface,

$$
N(T)=-2 N_{c} \int \frac{d^{3} k}{(2 \pi)^{3}} \frac{\partial n_{\mathbf{k}}}{\partial \epsilon_{\mathbf{k}}},
$$

and is simply written as

$$
N^{-1}(0)=\frac{\pi^{2}}{N_{c} k_{F}^{2}} v_{F}
$$

in the limit of zero temperature . Eq. (8) defines the Fermi velocity, which is given by the using the Lorentz transformation [8],

$$
\left.v_{F} \equiv \frac{\partial n_{\mathbf{k}}}{\partial \epsilon_{\mathbf{k}}}\right|_{|\mathbf{k}|=k_{F}}=\frac{k_{F}}{\mu}-\frac{N_{c} k_{F}^{2}}{3 \pi^{2}} f_{1}^{s},
$$

where $f_{1}^{s}$ is a spin-averaged Landau-Migdal parameter defined by

$$
f_{1}^{s}=\left.\frac{3}{4} \sum_{\zeta, \zeta^{\prime}} \int \frac{d \Omega_{\hat{\mathbf{k q}}}}{4 \pi} \cos \theta_{\hat{\mathbf{k q}}} f_{\mathbf{k}, \mathbf{q}}^{s}\right|_{|\mathbf{k}|=|\mathbf{q}|=k_{F}},
$$

with the relative angle $\theta_{\hat{\mathbf{k q}}}$ of $\mathbf{k}$ and $\mathbf{q}$.

Finally the magnetic susceptibility at zero temperature can be written in terms of the Landau-Migdal parameters,

$$
\chi_{M}=\chi_{\text {Pauli }}\left[1+\frac{N_{c} k_{F} \mu}{\pi^{2}}\left(-\frac{1}{3} f_{1}^{s}+\bar{f}^{a}\right)\right]^{-1},
$$

where $\chi_{\text {Pauli }}$ is the usual one for the Pauli paramagnetism, $\chi_{\text {Pauli }}=\bar{g}_{D}^{2} \mu_{q}^{2} N_{c} k_{F} \mu / 4 \pi^{2}$. 
When we consider the color-symmetric forward scattering amplitude of the two quarks around the Fermi surface by the one gluon exchange interaction (OGE), the direct term should be vanished due to the color neutrality of quark matter and the Fock exchange term gives a leading contribution. The color-symmetric OGE interaction of quasi-particles may be written,

$$
f_{\mathbf{k} \zeta, \mathbf{q} \zeta^{\prime}}=\frac{1}{N_{c}^{2}} \sum_{a, b} f_{\mathbf{k} \zeta a, \mathbf{q} \zeta^{\prime} b}=\frac{m}{E_{k}} \frac{m}{E_{q}} M_{\mathbf{k} \zeta, \mathbf{q} \zeta^{\prime}},
$$

with the invariant matrix element,

$$
M_{\mathbf{k} \zeta, \mathbf{q} \zeta^{\prime}}=-g^{2} \frac{1}{N_{c}^{2}} \operatorname{tr}\left(\lambda_{a} / 2 \lambda_{a} / 2\right) M^{\mu \nu}\left(k, \zeta ; q, \zeta^{\prime}\right) D_{\mu \nu}(k-q),
$$

where $M^{\mu \nu}\left(k, \zeta ; q, \zeta^{\prime}\right)=1 /\left(4 m^{2}\right) \operatorname{tr}\left[\gamma^{\mu}(\not k+m) P(a(\mathbf{k})) \gamma^{\nu}(\not q+m) P(a(\mathbf{q}))\right] ; P(a)$ is the projection operator to select a state with spin vector $a^{\mu}[2,3]$. It has been well known that massless gluons often causes infrared divergences in the Landau parameters [8, 10].

Since the one gluon exchange interaction is a long-range force and we consider the small energy-momentum transfer between quasi-particles, we must treat the gluon propagator by taking into account the hard-dense-loop (HDL) resummation [9]. Thus we take into account the screening effect for gluons,

$$
D_{\mu \nu}(k-q)=P_{\mu \nu}^{T} D_{T}(p)+P_{\mu \nu}^{L} D_{L}(p)-\xi \frac{p_{\mu} p_{\nu}}{p^{4}}
$$

with $p=k-q$, where $P_{\mu \nu}^{T(L)}$ is the standard projection operator onto the transverse (longitudinal) mode [9], which propagator has the form $D_{T(L)}(p)=$ $\left(p^{2}-\Pi_{T(L)}(p)\right)^{-1}$ in terms of the self-energy $\Pi_{T(L)}(p)$. The last term represents the gauge dependence with a parameter $\xi$. First of all we can easily check the gauge independence of the interaction between quasi-particles. Since $p_{\mu} p_{\nu} M^{\mu \nu}=0$ for the on-shell particles, any physical quantity described by the quasi-particle interaction is independent of the gauge parameter $\xi$ as should be.

The self-energies for the transverse and longitudinal gluons are given as

$$
\begin{aligned}
& \Pi_{L}\left(p_{0}, \mathbf{p}\right)=m_{D}^{2} \\
& \Pi_{T}\left(p_{0}, \mathbf{p}\right)=-i \frac{\pi u_{F} m_{D}^{2}}{4} \frac{p_{0}}{|\mathbf{p}|}, \quad u_{F} \equiv \frac{k_{F}}{E_{F}},
\end{aligned}
$$


in the limit $p_{0} /|\mathbf{p}| \rightarrow 0$, with the Debye screening mass, $m_{D}^{2} \equiv \sum_{f=u, d, s}\left(1 / 2 \pi^{2}\right) g^{2} \mu_{f} k_{F}^{f}$ [9]. Thus the longitudinal gluon is screened to have the Debye mass $m_{D}$, while the transverse gluon receives only the dynamic screening and the Fermi-liquid interaction on the Fermi surface is not screened. Then the quasiparticle interaction on the Fermi surface can be written as

$$
\left.f_{\mathbf{k} \zeta, \mathbf{q} \zeta^{\prime}}\right|_{|\mathbf{k}|=|\mathbf{q}|=k_{F}}=-C_{g} \frac{m^{2}}{E_{F}^{2}}\left[-M^{00} D_{L}(\mathbf{k}-\mathbf{q})+M^{i i} D_{T}(\mathbf{k}-\mathbf{q})\right]
$$

with the effective coupling strength, $C_{g}=\frac{N_{c}^{2}-1}{2 N_{c}^{2}} g^{2}$.

We can see that the both Landau parameters $f_{1}^{s}, \bar{f}^{a}$ include the infrared singularities due to the absence of the static screening for the transverse gluons; $D_{T}(\mathbf{k}-\mathbf{q}) \sim-1 /(\mathbf{k}-\mathbf{q})^{2}=-1 / 2 k_{F}^{2}\left(1-\cos \theta_{\hat{\mathbf{k q}}}\right)$ in this case, so that the logarithmic divergences appear in the Landau parameters through the integral over the relative angle, $\int d \Omega_{\hat{\mathbf{k q}}} 1 /\left(1-\cos \theta_{\hat{\mathbf{k q}}}\right)$.

Finally magnetic susceptibility is given as a sum of the contributions of the bare interaction and the static screening effect. We can see that the logarithmic divergences exactly cancel each other to give a finite result for susceptibility.

$$
\left(\chi_{M} / \chi_{\text {Pauli }}\right)^{-1}=1-\frac{C_{g} N_{c} \mu}{12 \pi^{2} E_{F}^{2} k_{F}}\left[m\left(2 E_{F}+m\right)-\frac{1}{2}\left(E_{F}^{2}+4 E_{F} m-2 m^{2}\right) \kappa \ln \frac{2}{\kappa}\right],
$$

with $\kappa=m_{D}^{2} / 2 k_{F}^{2}$. Obviously this expression is reduced to the simple OGE case without screening in the limit $\kappa \rightarrow 0$; one can see that the interaction among massless quarks gives a null contribution for the magnetic transition. The effect of the static screening for the longitudinal gluons gives the contribution of $g^{4} \ln \left(1 / g^{2}\right)$. In the nonrelativistic limit, it recovers the corresponding term in the RPA calculation of electron gas [4, 13].

To demonstrate the screening effect, we plot in Fig. 1 the magnetic susceptibility. We assume a flavor-symmetric quark matter, $\rho_{u}=\rho_{d}=\rho_{s}=\rho_{B} / 3$, and take the QCD coupling constant as $\alpha_{c} \equiv g^{2} / 4 \pi=2.2$ from the MIT bag model, where the value of $\alpha_{c}$ is chosen so as to reproduce the mass difference among different spin states. Since we can see from Eq. (17) that massless quarks, $u$ and $d$ quarks, never lead to magnetic phase transition, we only consider the magnetic susceptibility of massive quarks, $s$ quark with mass of $300 \mathrm{MeV}$ in the MIT bag model. Note that all the flavors always take part in the Debye mass, even if we are only concerned with the magnetic susceptibility of $s$ quarks. 


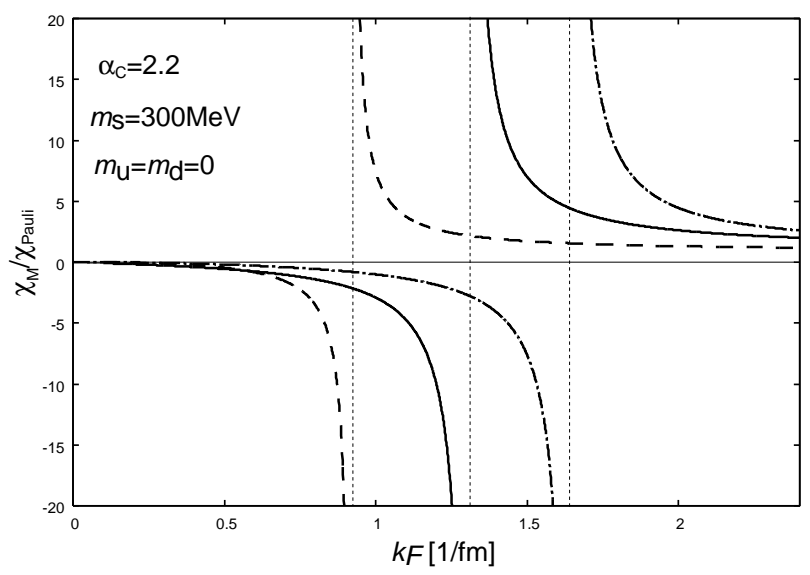

Figure 1: Magnetic susceptibility at $T=0$. Screening effects are shown in comparison with the simple OGE case: the solid curve shows the result with the simple OGE without screening, while the dashed and dash-dotted ones shows the screening effect with $N_{f}=1$ (only $s$ quark)and $N_{f}=2+1(u, d, s$ quarks), respectively.

One can see that the magnetic susceptibility diverges around $k_{F}=1 \sim$ $1.5 \mathrm{fm}^{-1}$ corresponding to order of nuclear density, where spontaneous magnetization appears. The critical density for the simple OGE without screening is consistent with the previous one given by the energy calculation [2]. One may expect that the quasi-particle interaction is effectively cut off at momentum $|\mathbf{k}|=m_{D}$, which reduces the strength of the Fock exchange interaction, once the screening is taken into account. However, this is not necessarily the case in QCD. Compare the screening effects by changing the number of flavors $\left(N_{f}\right)$; if we change $N_{f}$, the screening effect exhibits the opposite behavior for $N_{f}=1$ (only $s$ quark) and $N_{F}=2+1$ (u,d,s quarks). In the case of $N_{f}=1$ the screening effect works against the magnetic phase transition as in QED [4, while it favors in the case of $N_{f}=2+1$. This occurs due to the sign change of the logarithmic term in Eq. (17) at $\kappa=2$ (see Fig. 2): if $\alpha_{c} N_{f}>2 \pi, \kappa>2$ and the screening effect acts for the magnetic instability. 3 Hence we may say that the screening effect does not necessarily work

\footnotetext{
${ }^{3}$ It would be interesting to consider the criterion in the large $N_{c}$ and $N_{f}$ limits [11; since $\alpha_{c} N_{f} \sim O\left(N_{f} / N_{c}\right)$, the screening effect changes its property, depending on the ratio $N_{f} / N_{c}$.
} 
against the magnetic instability in QCD due to the large coupling constant and flavor degree of freedom.

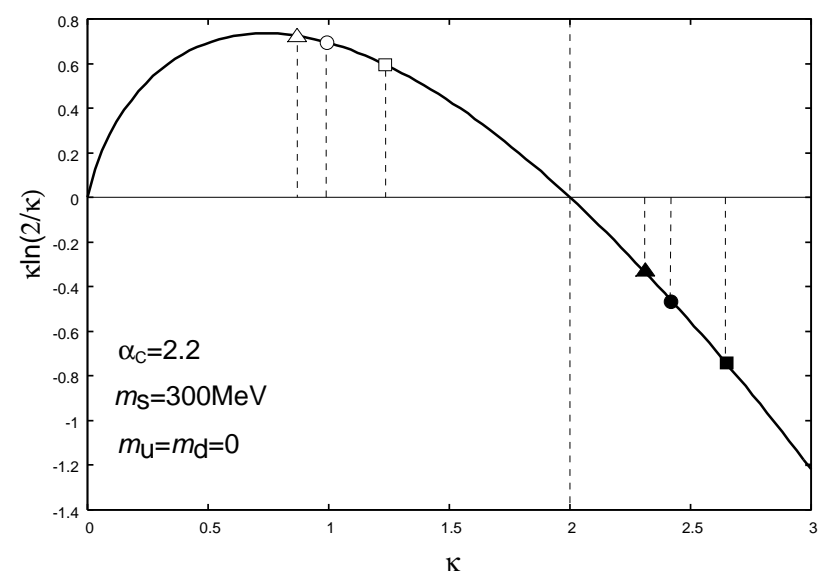

Figure 2: Behavior of the logarithmic function is sketched as a function of the ratio, $\kappa=m_{D}^{2} / 2 k_{F}^{2}$. Empty (full) symbols denote the case of $N_{f}=1(2+1)$ at $1.0,1.5,2.0 \mathrm{fm}^{-1}$ for square, circle, triangle, respectively. $\kappa=2$ is the point characterizing the change of the qualitative behavior of susceptibility; $\kappa$ for $N_{f}=1$ and $N_{f}=2+1$ lie in the different sides.

We have discussed the properties of the magnetic phase transition in QCD, by examining the magnetic susceptibility. The interesting behavior of the screening effect has been demonstrated, but it comes from the static screening for the longitudinal gluons. There is no static screening for the transverse gluons and the dynamic screening never contributes to the magnetic phase transition at $T=0$ due to the sharpness of the Fermi surface. However, one may expect that the dynamic screening gives a finite contribution due to the diffuseness of the Fermi surface. Actually it is well known that the non-Fermi-liquid effect gives an anomalous term $\propto T \ln T$ to the specific heat, which is responsible to the dynamic screening [12. We will consider the susceptibility in the finite temperature and discuss this issue in another paper [14].

This work is partially supported by the Grant-in-Aid for the 21st Century COE "Center for the Diversity and Universality in Physics" and the Grantin-Aid for Scientific Research Fund of the Ministry of Education, Culture, Sports, Science and Technology of Japan (13640282, 16540246). 


\section{References}

[1] T. Schäfer, hep-ph/0509068.

[2] T. Tatsumi, Phys. Lett. B489 (2000) 280.

T. Tatsumi, E. Nakano and K. Nawa, Dark Matter, p.39 (Nova Science Pub., New York, 2006).

[3] E. Nakano, T. Maruyama and T. Tatsumi, Phys. Rev. D68 (2003) 105001.

T. Tatsumi, E. Nakano and T. Maruyama, Prog. Theor. Phys. Suppl. 153 (2004) 190.

T. Tatsumi, T. Maruyama and E. Nakano, Superdense QCD Matter and Compact Stars, p.241 (Springer, 2006).

[4] C. Herring, Exchange Interactions among Itinerant Electrons: Magnetism IV (Academic press, New York, 1966)

K. Yoshida, Theory of magnetism (Springer, Berlin, 1998).

[5] A. Niegawa, Prog. Theor. Phys. 113 (2005) 581.

[6] A.K. Harding and D. Lai, Rep. Prog. Phys, 69 (2006) 2631,

[7] G. Baym and C.J. Pethick, Landau Fermi-Liquid Theory (WILEY-VCH, 2004)

P. Noziéres, Theory of Interacting Fermi Systems (Westview Press,1997).

[8] G. Baym and S.A. Chin, Nucl. Phys. A262 (1976) 527.

[9] J.I. Kapusta, Finite-temperature field theory (Cambridge U. Press, 1993).

M. Le Bellac, Thermal Field Theory (Cambridge U. Press, 1996).

T. Schäfer and F. Wilczek, Phys. Rev. D60 (1999) 114033.

[10] T. Tatsumi, arXiv:07113349.

[11] K. Ohnishi, M. Oka, S. Yasui, Phys. Rev. D76 (2007) 097501.

[12] A. Gerhold, A. Ipp and A. Rebhan, Phys. Rev. D70 (2004) 105015 and references cited therein.

T. Schäfer and Schwenzer, Phys. Rev. D70 (2004) 054007;114037. 
[13] K.A. Brueckner and K. Sawada, Phys. Rev. 112 (1957) 328.

B.S. Shastray, Phys. Rev. Lett. 38 (1977) 449.

[14] K. Sato and T. Tatsumi, (2008) in preparation. 